\title{
Complete protection for mice conferred by a DNA vaccine based on Japanese encephalitis virus P3 strain that is used to prepare the inactivated vaccine in China
}

Ran Wang ( $\nabla$ randall@mail.ccmu.edu.cn )

Beijing Children's Hospital https://orcid.org/0000-0002-0243-8538

Xiaozheng Yu

Capital Medical University

\section{Yan Wang}

Capital Medical University Affiliated Beijing Friendship Hospital

Xiaoyan Zheng

Capital Medical University Affiliated Beijing Friendship Hospital

\section{Research}

Keywords: Japanese encephalitis virus, P3, PrM/E, DNA vaccine, protection

Posted Date: January 7th, 2020

DOI: https://doi.org/10.21203/rs.2.20138/v1

License: (c) (i) This work is licensed under a Creative Commons Attribution 4.0 International License.

Read Full License 


\section{Abstract}

Background The incidence of Japanese encephalitis (JE) has been dramatically reduced in China after the coverage of the vaccine. It is believed that the live-attenuated Japanese encephalitis virus (JEV) vaccine SA14-14-2 has contributed a lot. Another vaccine that seems to have faded out of the public is an inactivated vaccine based on the JEV P3 strain, which is still considered to have certain modifiability, such as being transformed into a DNA vaccine to improve its immunogenicity.

Methods In this study, the protective efficacy induced by a Japanese encephalitis DNA vaccine candidate pV-JP3ME encoding pre-membrane (prM) and envelope (E) proteins of P3 strain in BALB/c mice. The $\mathrm{prM} / \mathrm{E}$ genes of the JEV P3 strain were subcloned into vector pVAX1 ( $\mathrm{pV}$ ) to construct pV-JP3ME.

Results The plasmid DNA was immunized BALB/c mice, high titers of IgG antibody and neutralizing antibody (nAb) against JEV were detected. The key cytokines in splenocytes upon stimulation with JEV antigens were secreted. Finally, complete protective efficacy was generated after challenge with the JEV P3 strain in mice.

Conclusions The DNA vaccine pV-JP3ME based on JEV P3 strain in this study can induce specific humoral immune and cytokine responses in mice, and provide complete protection for mice against JEV.

\section{Background}

Japanese encephalitis virus (JEV) is a single positive-strand RNA virus belonging to the Flaviviridae genus Flaviviridae [1]. JEV infection can cause severe encephalitis, neurological sequelae, and even death in children and adults [2]. JEV was first isolated in China in 1940, and it was transmitted mainly by Culex mosquito bites, with swine and wintering waterfowl as amplification hosts [3]. As of the end of the 1980s, JEV infection has always been a serious threat to the health of many Asian children, and nearly half of cases have occurred in China. Since the two types of vaccinations in China, the inactivated vaccine P3 strain and the live attenuated vaccine SA14-14-2 strain [4, 5], the incidence of Japanese encephalitis has decreased from 20.92/100,000 in 1971 to $0.12 / 100,000$ in 2011 [6, 7]. China is still using the two vaccines mentioned above, of which live-attenuated vaccines were included in the national Expanded Programme on Immunization (EPI) at the end of 2007 [8]. Given the reasons for the times of immunization and relatively low immunogenicity of inactivated vaccines, live-attenuated vaccines have now basically replaced inactivated vaccines [9]. Populations in some Asian countries and regions such as Japan, South Korea, Taiwan, and certain populations including immunocompromised or psychologically resistant vaccines in China, are still using inactivated vaccines [10]. Wild P3 strain has strong virulence and contains many key epitopes of immunogenicity, which can be improved by biological modification. In this study, by combining the biological characteristics of the virus and the advantages of the DNA vaccine, the $\mathrm{prM} / \mathrm{E}$ genes of the P3 strain was subcloned into the DNA vaccine vector pVAX1 $(\mathrm{pV})$ to obtain the JEV DNA vaccine pV-JP3ME. The comprehensive immune response and protection induced by it were evaluated and this study will provide important data for its further application. 


\section{Materials And Methods}

\section{Virus, cells, plasmid, and animal}

JEV (strain P3) was donated by Professor Min Cui from Huazhong Agricultural University and stored at $-80^{\circ} \mathrm{C}$. It was used as the coating antigen and stimulation for in vitro experiments and used for challenge experiments. Vero cells are used for plasmid transfection and plaque assay to detect virus titers, plaque reduction neutralization test (PRNT) are used to detect nAb titers. C6/36 cells are used for virus proliferation. The pV-JP3ME plasmid was constructed by introducing the $B a m H$ enzyme digestion site, Kozak sequence and signal sequence upstream of the prM/E sequence of P3 strain, and introducing Xhol digestion site downstream into the eukaryotic expression vector pV. Specific pathogen-free 6-8-week-old female BALB/c mice were purchased from Beijing Vital River Experimental Animal Technology Co., Ltd. and used for immunity, sera and splenocytes collection and challenge tests.

\section{Reagents and instruments}

Restriction enzymes BamH and Xhol, eukaryotic expression vector pV, nuclear staining agent 4',6diamidino-2-phenylindole (DAPI), and transfection reagent Lipofectamine 3000 were purchased from Thermo Scientific, USA. Minimal essential medium (MEM) and RPMI-1640 medium were purchased from Gibco, USA. Methylcellulose was purchased from Sigma, USA. Goat anti-mouse fluorescein isothiocyanate (FITC)-IgG antibody was purchased from Beijing TransGen Biotech, China. Goat antimouse horseradish peroxidase (HRP)-IgG antibody was purchased from Abcam, USA.

Tetramethylbenzidine (TMB) substrate color solution was purchased from MabTech company, USA. Enzyme-Linked ImmunoSpot (ELISPOT) kit, streptavidin and AEC color development kit were purchased from BD company, USA. The gene introduction instrument was purchased from Shanghai Teresa Corporation, China. The enzyme-linked immune-sorbent assay (ELISA) plate reader and cell culture incubator were purchased from Thermo, USA. The ELISPOT plate reader was purchased from CTL, USA.

\section{Transfection and immunofluorescence experiments}

pV-JP3ME or pV were transfected into Vero, respectively. After $5 \mathrm{~h}$, the transfection plasmid/reagent mixture was discarded and replaced with a complete culture medium. After $40 \mathrm{~h}$, the medium was discarded, and the two groups of cells were simultaneously fixed. Then, cells were incubated with JEV antiserum $(1: 1,000)$ as primary antibody and goat anti-mouse FITC-IgG as a secondary antibody. Observation of specific green fluorescence under a fluorescence microscope indicates that the plasmid was successfully transfected and expressed in mammalian cells in vitro.

\section{Animal experiments}

The mice were randomly divided into two groups. The vaccine group was vaccinated with the DNA vaccine $\mathrm{pV}$-JP3ME, and the control group was vaccinated with the empty vector plasmid $\mathrm{pV}$. Immunization was performed three times, each immunization dose was $50 \mu \mathrm{g}$, intramuscular injection with electroporation was used. 1 and 3 weeks after the last immunization, the splenocytes and sera of the 
two groups of mice were taken, respectively. The next day, mice in the vaccine and the control group were challenged with JEV. The body weight changes and survival rate were measured daily after the challenge, and the observation was for 12 consecutive days. The animal experiment schedule was showed in Fig. 1.

\section{Plaque assay}

Vero cells were cultured into 24-well plates, and the cell density must be more than $95 \%$ before performing the virus infection. The serially diluted virus was serially 10 -fold-diluted from the original solution (1:1), for a total of seven dilutions, namely $1: 1$ to $1: 10^{6} .200 \mu \mathrm{L}$ of the virus dilution was added to each well and incubated at $37^{\circ} \mathrm{C}$ for $1 \mathrm{~h}$. Gently shake the plate once every $15 \mathrm{~min}$. After discarding the dilution, add $5 \mathrm{~mL}$ of MEM medium containing 1.2\% methylcellulose to each well. After $4 \mathrm{~d}$ of culture, to discard the medium and add $1 \mathrm{~mL}$ of crystal violet staining solution to each well and stain for $30 \mathrm{~min}$ at room temperature. The number of plaques per well was counted and the virus titer was calculated. The titer of the virus solution needs to be repeatedly measured three times and an average value is taken, which is expressed as a plaque-forming unit (PFU)/mL.

\section{ELISA}

Heat-inactivated JEV was coated into a 96 -well plate at $10^{5} \mathrm{PFU}$ per well at $4{ }^{\circ} \mathrm{C}$ overnight. The coating antigen was discarded and blocked with $1 \%$ bovine serum albumin (BSA) at $37^{\circ} \mathrm{C}$ for $2 \mathrm{~h}$. The blocking solution was discarded. The sera of each group of mice were started from 1:100, and was serially diluted at a two-fold ratio, for a total of 12 dilutions, namely from 1:100 to 1:204,800, and added to the wells in turn as a primary antibody, $4{ }^{\circ} \mathrm{C}$ overnight. The next day, the primary antibody was discarded. After washing the plate five times, HRP-labeled goat anti-mouse IgG antibody $(1: 4,000)$ was added as a secondary antibody. After incubation at $37^{\circ} \mathrm{C}$ for $1 \mathrm{~h}$, the plate was discarded. The substrate solution developed color for $20 \mathrm{~min}$, and the reaction was stopped with $\mathrm{H}_{2} \mathrm{SO}_{4}$. To take $1 / 2$ of the $\mathrm{A}_{450} \mathrm{~nm}$ value at the 1:100 dilution of the control group as the cut-off value, and the maximum dilution greater than this cut-off value is the serum IgG antibody titer.

\section{PRNT}

Vero cells were cultured into 24 -well plates as described previously [11, 12]. The serum is diluted from $1: 10$, and is serially diluted at a 2-fold ratio. There are seven consecutive dilutions, that is, 1:10 to 1:640. Each dilution of serum is mixed with an equal volume of virus solution (containing $100 \mathrm{PFU}$ ). To incubate at $37^{\circ} \mathrm{C}$ for $1 \mathrm{~h}$, and set serum-free virus samples at $4{ }^{\circ} \mathrm{C}$ and $37^{\circ} \mathrm{C}$, respectively, for excluding temperature factor and referencing positive virus count. Then, the serum/virus mixture was added to the wells in order and incubated at $37^{\circ} \mathrm{C}$ for $1 \mathrm{~h}$. During the period, the plate was shaken gently every 15 min. The subsequent step is the same as described in 2.5. The serum dilution corresponding to a $50 \%$ reduction in the number of plaques in the positive wells incubated at $37^{\circ} \mathrm{C}$ was recorded as the PRNT 50 value, which is the nAb titer.

\section{ELISPOT assay}


IL-2 and IFN- $y$ capture antibody diluted 1:200 were coated in a 96-well plate at $4{ }^{\circ} \mathrm{C}$ overnight. To discard the coating solution and block with RPMI-1640 medium containing 10\% FBS at room temperature for $2 \mathrm{~h}$. Then, splenocytes of two groups were added to each well, $2 \times 10^{5}$ per well, and $10^{5} \mathrm{PFU}$ heat-inactivated JEV was added as a stimulus, and cultured at $37^{\circ} \mathrm{C}$ for $72 \mathrm{~h}$. After discarding the cultured splenocytes, IL2 and IFN- $y$ detection antibodies were added, respectively, and then spot forming unit (SFU) was counted by adding streptavidin and AEC chromogenic solution.

\section{Statistical analysis}

All experimental data were recorded using Excel 2016 software, statistical analysis was performed using SPSS 17.0 software (USA), body weight change was analyzed by repeated analysis of multivariate analysis of variance, survival rates were compared using Log-rank test, and the differences between the other groups were compared using one-way ANOVA analysis, quantitative data are expressed as mean \pm standard deviation. $P<0.05$ was considered statistically significant.

\section{Results}

\section{Target protein prM/E expression in vitro}

As shown in Fig. 2, after Vero cells were transfected with pV-JP3ME, expressed target protein could bind to JEV anti-sera and show specific green fluorescence, while Vero cells transfected with empty vector plasmid $\mathrm{pV}$ showed no specific fluorescence, indicating that the target protein $\mathrm{prM} / \mathrm{E}$ can successfully be expressed in eukaryotic cells and has reactogenicity, which can be used for subsequent experimental research.

\section{IgG antibody levels}

As shown in Fig. 3a, three weeks after the last immunization, the sera of the vaccine group and the control group were collected, and the titer of the anti-JEV-specific IgG antibodies was measured by ELISA. Higher IgG antibodies against JEV were detected in the sera of mice in the vaccine group than in the control group, with a titer of 1:4,200, compared with 1:163 in the control group $(P<0.001)$. There is a statistical difference between them, as shown in Fig. 3a, indicating that after immunization with the JEV DNA vaccine $\mathrm{pV}$-JP3ME, mice can induce high levels of IgG antibodies against JEV.

\section{NAb levels}

The titer of anti-JEV-specific nAb was measured by PRNT. The results showed that the nAb titer against $J E V$ in the sera of the vaccine group was 1:380, and that in the control group was 1:11 $(P<0.001)$. There is a statistical difference between the groups, as shown in Fig. 3b, suggesting that after the pV-JP3MEimmunization, mouse sera had a neutralizing activity against JEV.

\section{Cytokine-producing levels}


One week after the last immunization, splenocytes of the vaccine group and the control group were obtained, and ELISPOT was used to detect the IL-2- and IFN-y-spots of splenocytes secreted upon stimulation with the JEV antigen. The results showed that the spots of IL-2 and IFN- $\gamma$ were significantly greater in the splenocytes of the vaccine group than with the control group in vitro, the differences were statistically significant $(P<0.01)$, as shown in Figure $3 \mathrm{c}$ and $3 \mathrm{~d}$. After mice were immunized with pVJP3ME, splenocytes could secrete higher levels of IL-2 and IFN- $\gamma$ after stimulation with JEV antigen, suggesting a better antiviral cytokine response.

\section{Protective effects}

In order to evaluate the protective efficacy of the JEV DNA vaccine pV-JP3ME in mice, $1 \times 10^{5}$ PFU JEV was used to challenge the mice in the vaccine and the control group intraperitoneally, and the change in body weight and survival rate were recorded and compared for 12 consecutive days. After the challenge with JEV, the body weight change of vaccinated mice showed a steady trend during the observation period, and the differences between individuals were limited, while that of the control mice continued to decline. A statistical difference was shown between the two groups $(P<0.01$, Fig. 4a). In terms of survival rate, mice in the vaccine group were fully protected, with a survival rate of $100 \%(8 / 8)$, while mice in the control group began to die on the sixth day, and all died on the twelfth day $(0 / 8)$. The difference between the groups is statistically significant $(P<0.001$, Fig. $4 \mathrm{~b})$.

\section{Discussion}

Currently, the JEV vaccine widely used in China is the live attenuated vaccine SA14-14-2 strain. This vaccine has a good immune effect. In addition to China, there are still many Asian countries that approved it for use. At the same time, another self-developed inactivated vaccine P3 strain in China is still in use. Most of the vaccinees in China are those with low immune function or defects and some special populations (psychological factors of excluding live vaccines or unsafe vaccine incidents in society). However, the P3 inactivated vaccine rather than the SA14-14-2 vaccine is not included in the EPI, the former one requires full payment at its own expense, but it still has a solid market share as described above. In some Asian countries and regions, such as South Korea, Japan, and Taiwan, inactivated vaccines are customary and considered to be safe. Most of the inactivated vaccines they used are Nakayama strain [13], which originated from Japan and still predominantly occupy the JEV vaccine market, although different types of live attenuated vaccines have been introduced into these regions. Inactivated vaccine does have some disadvantages that cannot be compared with live-attenuated vaccines, such as low immunogenicity, significant Th2-typed immune tendency, multiple immunizations, etc [14]. But in terms of its safety, inactivated vaccines do not have virulence recovery and reproducibility, which still makes it highly respected in many JE-effected countries. After all, the vaccinated population is mainly children around the age of one-year-old, and safety cannot be ignored.

It is well known that the SA14-14-2 live-attenuated vaccine requires only two injections, while the inactivated P3 vaccine requires four or more vaccination to achieve sufficient protection. This greatly 
limits the possibility that the vaccine will become the first choice for travelers' emergency vaccines. Even in JE-endemic countries like China, it is not admired for comparison. It is difficult to develop a live attenuated vaccine based on the P3 strain, and even if it is successful, it is difficult to compete with the existing SA14-14-2 strain, because the latter has been certified and recommended by the World Health Organization. In comparison, DNA vaccines have been used in the prevention and treatment of multiple pathogens and diseases in recent years, such as infectious diseases such as dengue virus, human immunodeficiency virus, malaria, and tumor vaccines. Due to the balanced immune response and the long-lasting effect induced by the DNA vaccine, it has become a popular alternative.

Therefore, in this study, the most antigenic structural protein prM/E of P3 strain JEV was used as a target to construct a DNA vaccine. First, we verified the success or failure of expressing the target antigen in the transfection experiments of mammalian cells in vitro. The vaccine expresses the target protein which can stably bind to JEV-specific antibodies, suggesting that it has good reactivity. Regarding the route, time and dose of immunizations, we have already fully studied other flavivirus vaccines, such as dengue virus and Zika virus, and will not discuss here.

After three immunizations with the vaccine, mice can induce high titer $\lg G$ antibodies and $n A b$ in vivo. At the same time, the splenocytes of immunized mice produced high levels of cytokine IL-2 and IFN- $\gamma$ upon the stimulation of JEV-specific antigen. IL-2 plays an important role in the maturation, proliferation, and activation of T cells. IFN- $\gamma$ is one of the most important innate and acquired antiviral cytokines, which can play an anti-viral utility in both innate and adaptive immunity [15]. We selected these two representative cytokines for testing, suggesting that the vaccine is well immunogenic. Furthermore, through the challenge test in vivo, pV-JP3ME provided complete protection for the mice, which made the mice resistant to the lethal dose of JEV, while the control group all died. The above results indicate that $\mathrm{prM} / \mathrm{E}$ protein of the P3 strain is sufficient as a target protein candidate of the JEV vaccine to induce an effective immunoprotective effect $[11,16]$.

In recent years, there has been a lot of researches on SA14-14-2, and the summary shows the scalability of the vaccine. This is indeed the case, Erra et al. prepared an inactivated vaccine by using the live attenuated vaccine SA14-14-2 strain, and on the other hand [17], Appaiahgari et al. replace the prM/E of the yellow fever virus attenuated strain 17D with that of SA14-14-2 by using chimeric recombination [18], they have also obtained a good immune effect. One of the reasons for selecting the P3 strain in this study is that the virulence of the P3 strain is closer to that of the wild strain than SA14-14-2, and it is presented in the form of a DNA vaccine to ensure that there is no possibility of virulence recovery, and expressed target protein is similar to the natural conformation of the original strain $\mathrm{prM} / \mathrm{E}$ protein containing key epitopes that are not displayed in the attenuated strain. The second is that P3 is an inactivated vaccine source strain widely used in China, the recombinant construction can be used in combination with the P3 inactivated vaccine in heterogeneous immunization in subsequent studies. Previous studies have reported that a DNA vaccine constructed using a certain target protein is used as the prime immunization, then a protein component vaccine (subunit vaccine) is prepared with the same target protein is boosted, the immune response is more robust than homologous immunization, namely 
DNA alone or subunit immunization alone [19]. The level of the immune response induced by heterologous immunization is higher, more balanced, and the protective effect is also improved. This idea is expected to reduce the times of immunization compared with immunizing an inactivated vaccine alone, and theoretically, it can also improve the immunogenicity and prolong the duration of the vaccine $[20,21]$, which warrants later experiments.

\section{Conclusions}

In summary, this study used the P3 strain to construct a JEV DNA vaccine candidate, evaluated its immunogenicity and protective effect in mice, and confirmed that the vaccine can induce JEV-specific humoral and cytokine responses in mice, and provide mice with complete protection against JEV. Our data will provide a basis for the subsequent promotion and use of the vaccine, and lay the foundation for its combined use with inactivated vaccines of the same strain in a heterologous regimen.

\section{Abbreviations}

BSA: Bovine serum albumin; DAPI: 4',6-diamidino-2-phenylindole; E: Envelope; ELISA: Enzyme-linked immune-sorbent assay; EPI: Expanded Programme on Immunization; ELISPOT: Enzyme-Linked ImmunoSpot; FITC: Fluorescein isothiocyanate; HRP: horseradish peroxidase; JE: Japanese encephalitis; JEV: Japanese encephalitis virus; MEM: Minimal essential medium; NAb: Neutralizing antibody; PFU: Plauqe-forming unit; PrM: Premembrane; PRNT: Plaque reduction neutralization test; $p V$ : pVAX1; SFU: Spot-forming unit; TMB: Tetramethylbenzidine.

\section{Declarations}

\section{Acknowledgements}

Not applicable.

\section{Authors' contributions}

RW designed and performed the experiments. $X Y$ and $Y W$ performed the experiments. $X Z$ analyzed the data and wrote the manuscript. All authors read and approved the final manuscript.

\section{Funding}

This work is supported by Research Foundation of Capital Medical University (PYZ2018052) and Startup Fund of Beijing Friendship Hospital, Capital Medical University (Yyqdkt2016-24).

\section{Availability of data and materials}

The datasets used during the current study are available from the corresponding author on reasonable request. 


\section{Ethics approval and consent to participate}

This work was approved by the Ethics Committee of Beijing Friendship Hospital, the Second Clinical Medical College of Capital Medical University.

\section{Consent for publication}

Not applicable.

\section{Competing interests}

The authors declare that they have no competing interests.

\section{Author details}

${ }^{1}$ Key Laboratory of Major Diseases in Children, Ministry of Education, National Clinical Research Center for Respiratory Diseases, Beijing Key Laboratory of Pediatric Respiratory Infection Diseases, Virology Laboratory, Beijing Pediatric Research Institute, Beijing Children's Hospital, Capital Medical University, National Center for Children's Health, Research Unit of Critical Infection in Children, Chinese Academy of Medical Sciences, 2019RU016, Beijing, 100045, China. ${ }^{2}$ Beijing Friendship Hospital, the Second Clinical Medical College of Capital Medical University, Beijing, 100050 China. ${ }^{3}$ Beijing Institute of Tropical Medicine, Beijing Key Laboratory for Research on Prevention and Treatment of Tropical Diseases, Beijing, 100050, China

\section{References}

1.

Gao X, Liu H, Li X, Fu S, Cao L, Shao N, Zhang W, Wang Q, Lu Z, Lei W, et al. Changing Geographic Distribution of Japanese Encephalitis Virus Genotypes, 1935-2017. Vector Borne Zoonotic Dis. 2019;19:35-44.

2.

Solomon T, Dung NM, Kneen R, Thao le TT, Gainsborough M, Nisalak A, Day NP, Kirkham FJ, Vaughn DW, Smith S, White NJ. Seizures and raised intracranial pressure in Vietnamese patients with Japanese encephalitis. Brain. 2002;125:1084-93.

3 .

Mansfield KL, Hernandez-Triana LM, Banyard AC, Fooks AR, Johnson N. Japanese encephalitis virus infection, diagnosis and control in domestic animals. Vet Microbiol. 2017;201:85-92.

4.

Liu X, Yu Y, Li M, Liang G, Wang H, Jia L, Dong G. Study on the protective efficacy of SA14-14-2 attenuated Japanese encephalitis against different JE virus isolates circulating in China. Vaccine. 2011;29:2127-30.

5. 
Wang HY, Liang GD. [Comparison of nucleotide and deduced amino acid sequences of E gene of the newly isolated Japanese encephalitis virus strains and inactivated vaccine strain P3]. Zhonghua Shi Yan He Lin Chuang Bing Du Xue Za Zhi. 2006;20:56-60.

6.

Zhang L, Luan RS, Jiang F, Rui LP, Liu M, Li YX, Yin ZD, Luo HM. Epidemiological characteristics of Japanese encephalitis in Guizhou Province, China, 1971-2009. Biomed Environ Sci. 2012;25:297-304. 7.

Gao X, Li X, Li M, Fu S, Wang H, Lu Z, Cao Y, He Y, Zhu W, Zhang T, et al. Vaccine strategies for the control and prevention of Japanese encephalitis in Mainland China, 1951-2011. PLoS Negl Trop Dis. 2014;8:e3015.

8.

Li X, Ma SJ, Liu X, Jiang LN, Zhou JH, Xiong YQ, Ding H, Chen Q. Immunogenicity and safety of currently available Japanese encephalitis vaccines: a systematic review. Hum Vaccin Immunother. 2014;10:357993.

9.

Yao Z, Dong G, Yu Y. [Study on a purified and inactivated Japanese encephalitis vaccine prepared on Vero cells using SA14-14-2 attenuated virus strain]. Zhonghua Shi Yan He Lin Chuang Bing Du Xue Za Zhi. 1999;13:191-3.

10.

Chen HL, Chang JK, Tang RB. Current recommendations for the Japanese encephalitis vaccine. J Chin Med Assoc. 2015;78:271-5.

11.

Wang R, Liao X, Fan D, Wang L, Song J, Feng K, Li M, Wang P, Chen H, An J. Maternal immunization with a DNA vaccine candidate elicits specific passive protection against post-natal Zika virus infection in immunocompetent BALB/c mice. Vaccine. 2018;36:3522-32.

12.

Wang R, Xie L, Gao N, Fan D, Chen H, Wang P, Zhou H, An J. Decreases in Both the Seroprevalence of Serum Antibodies and Seroprotection against Japanese Encephalitis Virus among Vaccinated Children. Virol Sin. 2019;34:243-52.

13.

Lee S, Moon H, Kim MG, Kim do K, Chung HJ, Park YK, Oh HJ. Establishment of the 3rd national standard for lot release testing of the Japanese encephalitis vaccine (Nakayama-NIH strain) in Korea. Hum Vaccin Immunother. 2016;12:1805-7.

14.

Zakhartchouk AN, Liu Q, Petric M, Babiuk LA. Augmentation of immune responses to SARS coronavirus by a combination of DNA and whole killed virus vaccines. Vaccine. 2005;23:4385-91.

15.

Paliard X, de Waal Malefijt R, Yssel H, Blanchard D, Chretien I, Abrams J, de Vries J, Spits H. Simultaneous production of IL-2, IL-4, and IFN-gamma by activated human CD4 + and CD8 + T cell clones. J Immunol. 1988;141:849-55. 
16.

Galula JU, Chang GJ, Chuang ST, Chao DY. Establishment of an Algorithm Using prM/E- and NS1-Specific IgM Antibody-Capture Enzyme-Linked Immunosorbent Assays in Diagnosis of Japanese Encephalitis Virus and West Nile Virus Infections in Humans. J Clin Microbiol. 2016;54:412-22.

17.

Erra EO, Kantele A. The Vero cell-derived, inactivated, SA14-14-2 strain-based vaccine (Ixiaro) for prevention of Japanese encephalitis. Expert Rev Vaccines. 2015;14:1167-79.

18.

Appaiahgari MB, Vrati S. IMOJEV((R)): a Yellow fever virus-based novel Japanese encephalitis vaccine. Expert Rev Vaccines. 2010;9:1371-84.

19.

Peng S, Qiu J, Yang A, Yang B, Jeang J, Wang JW, Chang YN, Brayton C, Roden RBS, Hung CF, Wu TC. Optimization of heterologous DNA-prime, protein boost regimens and site of vaccination to enhance therapeutic immunity against human papillomavirus-associated disease. Cell Biosci. 2016;6:16. 20.

Forster KM, Hartwig DD, Oliveira TL, Bacelo KL, Schuch R, Amaral MG, Dellagostin OA. DNA prime-protein boost based vaccination with a conserved region of leptospiral immunoglobulin-like $A$ and $B$ proteins enhances protection against leptospirosis. Mem Inst Oswaldo Cruz. 2015;110:989-95.

21.

Gupta S, Garg NJ. A Two-Component DNA-Prime/Protein-Boost Vaccination Strategy for Eliciting LongTerm, Protective T Cell Immunity against Trypanosoma cruzi. PLoS Pathog. 2015;11:e1004828.

\section{Figures}

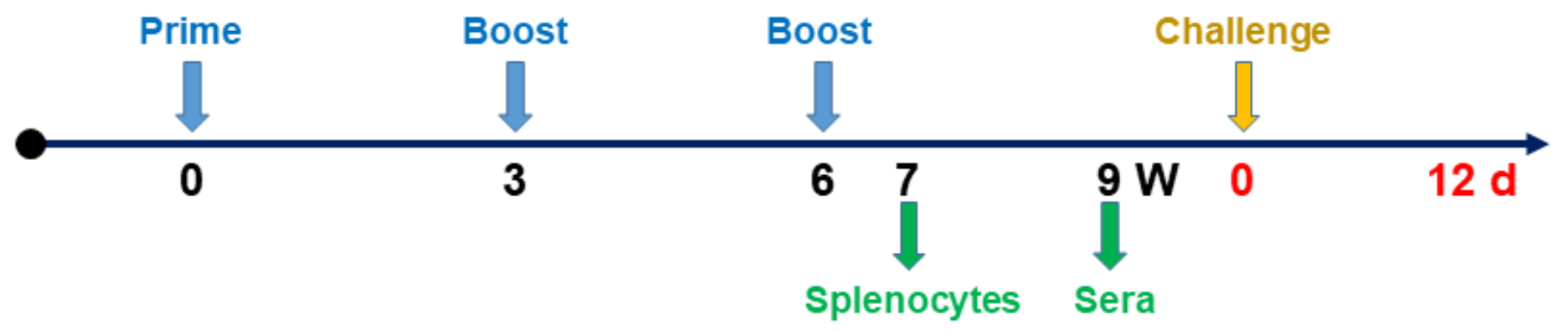

Figure 1

Mouse experimental workflow. Groups of mice were immunized by intramuscular electroporation with 50 $\mu \mathrm{g}$ of either $\mathrm{pV}$-JP3ME DNA vaccine or $\mathrm{pV}$ in each limb individually and were boosted twice at three-week intervals. Splenocytes were obtained one week after final immunization, and sera were collected three weeks after final immunization, respectively. Subsequently, vaccinated mice were challenged with $1 \times 105 P F U$ of JEV P3 strain. Body weight changes and survival rates were observed for 12 consecutive days after challenge. 

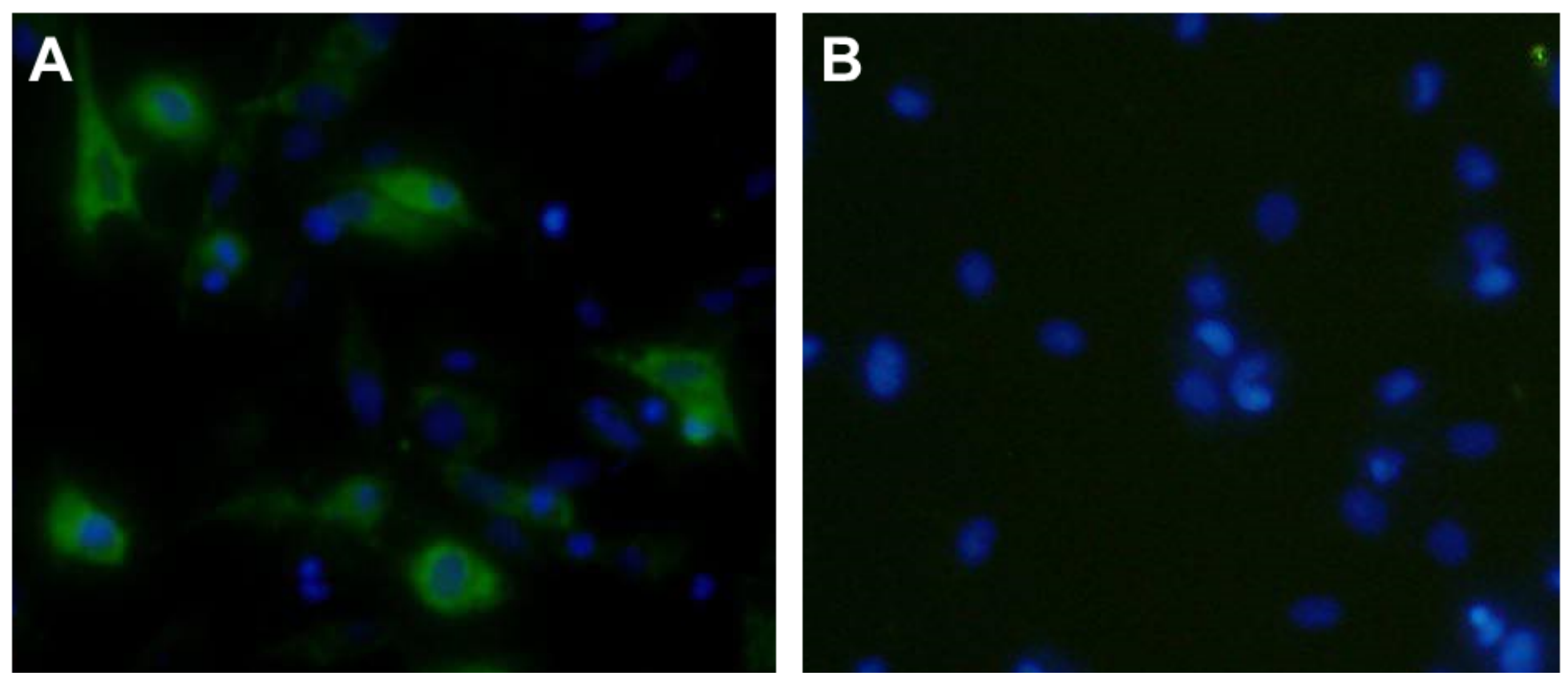

Figure 2

Representative images of immunofluorescence after Vero cells were transfected with plasmid DNA. After Vero cells were transfected with a pV-JP3ME or b pV, respectively, JEV anti-serum was used as the primary antibody, and goat anti-mouse FITC-IgG was used as the secondary antibody to stain. The left image shows specific green fluorescence, but the right image does not $(\times 200)$. 
A

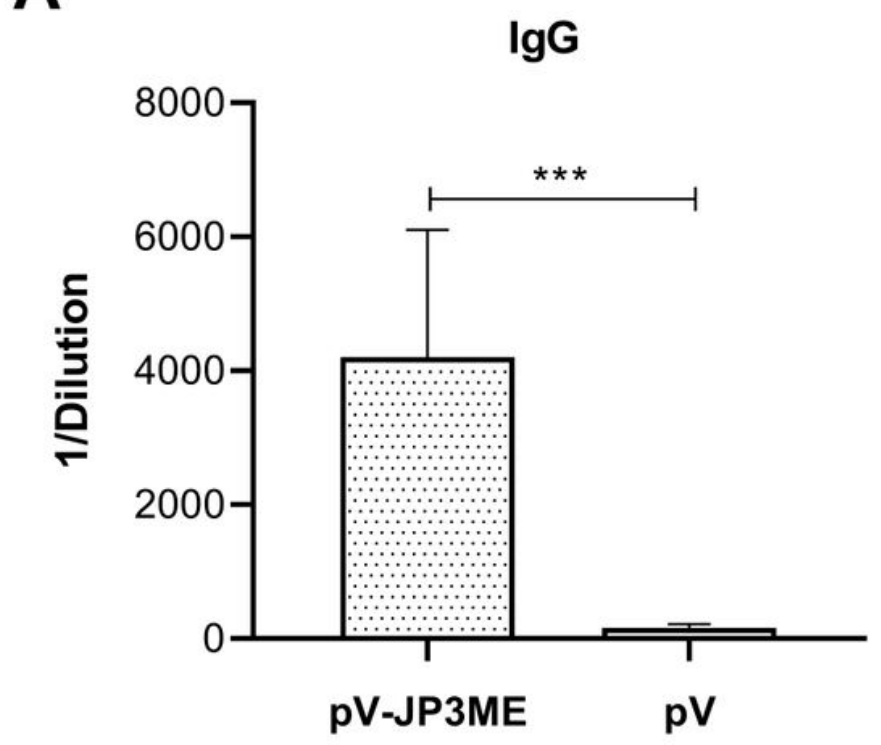

C

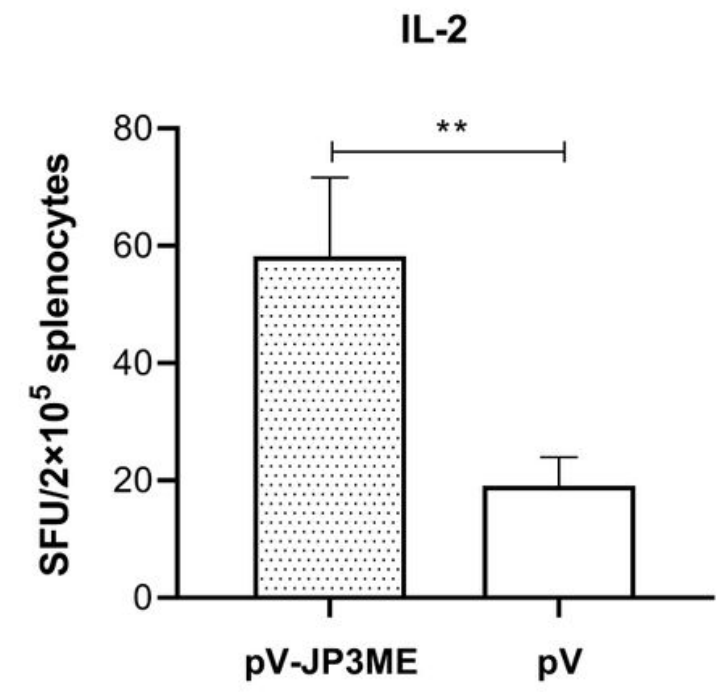

B

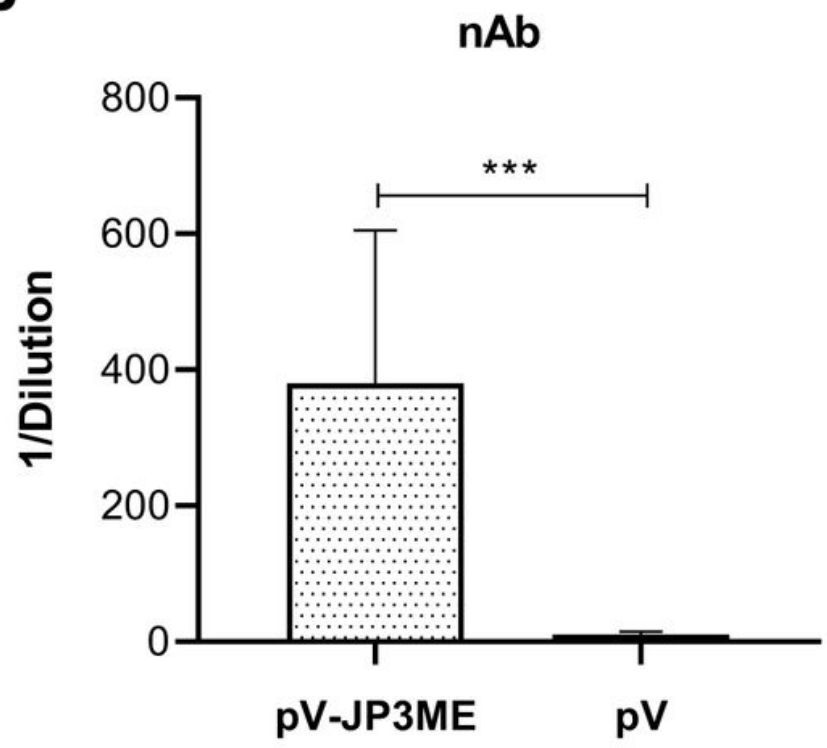

D

IFN-Y

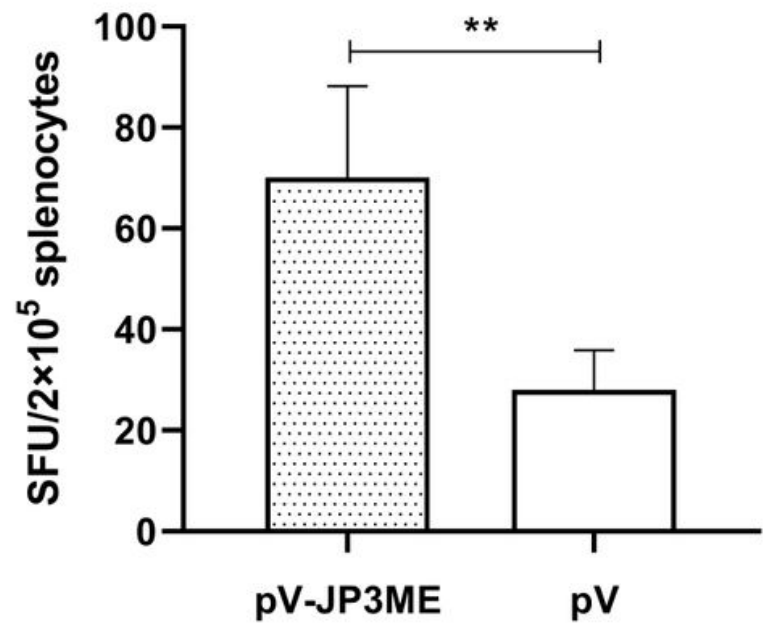

Figure 3

Specific humoral immune response and cytokine secretion produced by mice after immunization. In the third week after the mice were immunized three times with $\mathrm{pV}$-JP3ME or pV, a IgG antibodies and b nAb were produced with higher titers in the vaccine group than those in the control group. One week after immunization, mouse splenocytes produced high levels of c IL-2 and d IFN- $\gamma$ upon JEV antigen stimulation. ${ }^{*} \mathrm{P}<0.01,{ }^{* \star *} \mathrm{P}<0.001, \mathrm{n}=8$ per group. 
A

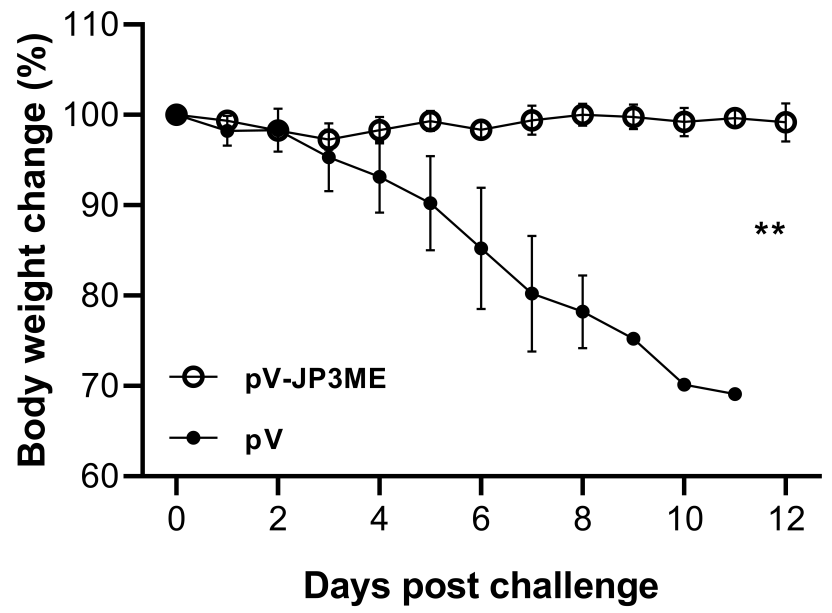

B

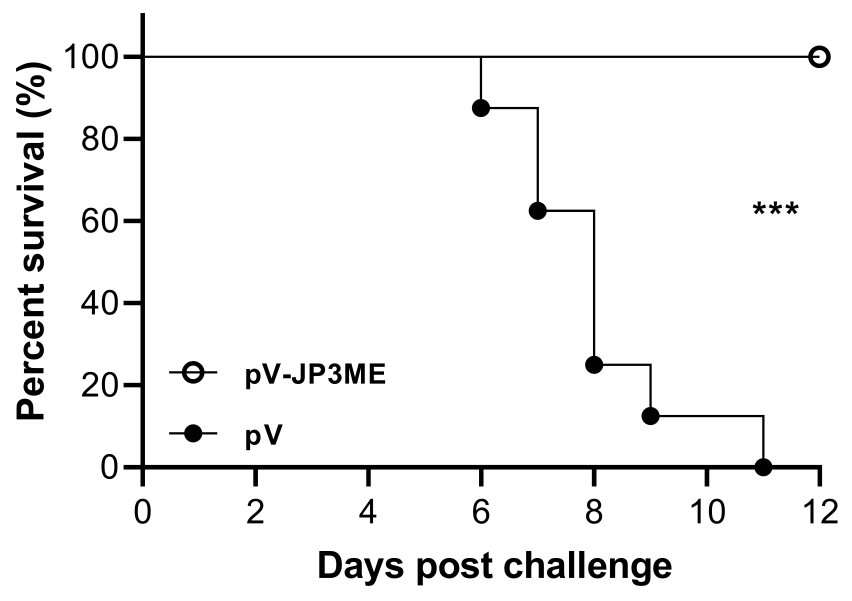

\section{Figure 4}

Protective effect generated by mice after immunization. In the third week after the mice were immunized with three pV-JP3ME or pV doses, the challenge test was performed. The mice in the vaccine group had no significant a body weight changes within 12 days, while the control group continued to decline. Similarly, the mice in the vaccine group were completely protection, $b$ the survival rate was up to $100 \%$, while the control group all died. ${ }^{\star *} \mathrm{P}<0.01,{ }^{\star}{ }^{*} \mathrm{P}<0.001, \mathrm{n}=8$ per group. 